\title{
Characterizing the Core via $k$-Core Covers
}

Estela Sánchez-Rodríguez ${ }^{1}$

Peter Borm ${ }^{2}$

Arantza Estévez-Fernández ${ }^{3}$

M. Gloria Fiestras-Janeiro'

Manuel A. Mosquera'

1 Vigo University, Spain;

2 CentER, Tilburg University, The Netherlands;

3 Faculty of Economics and Business Administration, VU University Amsterdam, and Tinbergen Institute, The Netherlands. 
Tinbergen Institute is the graduate school and research institute in economics of Erasmus University Rotterdam, the University of Amsterdam and VU University Amsterdam.

More TI discussion papers can be downloaded at http://www.tinbergen.nl

Tinbergen Institute has two locations:

Tinbergen Institute Amsterdam

Gustav Mahlerplein 117

1082 MS Amsterdam

The Netherlands

Tel.: +31(0)205251600

Tinbergen Institute Rotterdam

Burg. Oudlaan 50

3062 PA Rotterdam

The Netherlands

Tel.: +31(0)10 4088900

Fax: $+31(0) 104089031$

Duisenberg school of finance is a collaboration of the Dutch financial sector and universities, with the ambition to support innovative research and offer top quality academic education in core areas of finance.

DSF research papers can be downloaded at: http://www.dsf.nl/

Duisenberg school of finance

Gustav Mahlerplein 117

1082 MS Amsterdam

The Netherlands

Tel.: +31(0)20 5258579 


\title{
Characterizing the core via $k$-core covers*
}

\author{
E. Sánchez-Rodríguez ${ }^{1} \quad$ P. Borm ${ }^{2} \quad$ A. Estévez-Fernández ${ }^{3}$ \\ M.G. Fiestras-Janeiro ${ }^{1} \quad$ M.A. Mosquera ${ }^{1}$ \\ ${ }^{1}$ Department of Statistics and Operations Research, Vigo University, Spain. \\ ${ }^{2}$ CentER and Department of Econometrics and Operations Research, Tilburg University, The Netherlands. \\ 3 Tinbergen Institute and Department of Econometrics and Operations Research, VU University Amsterdam, The \\ Netherlands.
}

\begin{abstract}
This paper extends the notion of individual minimal rights for a transferable utility game (TU-game) to coalitional minimal rights using minimal balanced families of a specific type, thus defining a corresponding minimal rights game. It is shown that the core of a TU-game coincides with the core of the corresponding minimal rights game. Moreover, the paper introduces the notion of the $k$-core cover as an extension of the core cover. The $k$-core cover of a TU-game consists of all efficient payoff vectors for which the total joint payoff for any coalition of size at most $k$ is bounded from above by the value of this coalition in the corresponding dual game, and from below by the value of this coalition in the corresponding minimal rights game. It is shown that the core of a TU-game with player set $N$ coincides with the $\left\lfloor\frac{|N|}{2}\right\rfloor$-core cover. Furthermore, full characterizations of games for which a $k$-core cover is nonempty and for which a $k$-core cover coincides with the core are provided.
\end{abstract}

Keywords: Core; Core cover; $k$-core cover; $k$-compromise admissibility; $k$-compromise stability; Assignment games

\section{Introduction}

The core of a transferable utility game (TU-game), as introduced by Gillies (1953), consists of all efficient payoff vectors for the monetary value of the grand coalition from which no coalition has an incentive to deviate. Some well-known core catchers are the dominance core (cf. Gillies, 1953, 1959), the Weber set (Weber, 1988) and the core cover (Tijs and Lipperts, 1982).

The literature shows that both convex games (Shapley, 1971), for which the core equals the Weber set, and compromise stable games (Quant et al, 2005), which are balanced games for which the core equals the core cover, have several interesting and helpful properties. Restricting attention to compromise stability, we want to mention that the nucleolus (Schmeidler, 1969)

\footnotetext{
${ }^{*}$ Authors acknowledge the financial support of Ministerio de Ciencia, MTM2011-27731-C03. Corresponding author: esanchez@uvigo.es
} 
of any compromise stable game can be directly computed by using the Aumann Maschler rule (Aumann and Maschler, 1985) of an associated bankruptcy game (cf. O'Neill , 1982), thus unifying seemingly unrelated results on the nucleolus for, e.g., bankruptcy games and clan games (Potters et al, 1989).

The definition of the core cover of a TU-game is based on individual minimal rights and on individual marginal contributions to the grand coalition $N$. This paper aims for an extension of the core cover based on coalitional considerations. Individual marginal contributions can readily be extended to coalitional marginal contributions using the corresponding dual game. This paper proposes to extend individual minimal rights to coalitional ones by using for each coalition $S$ minimal balanced families on $N \backslash S$ with the size of its elements restricted to at most $|S|$. In this way, an associated minimal rights game is obtained. A first result shows that the core of a TU-game coincides with the core of its corresponding minimal rights game.

Using the dual game and the minimal rights game, we define the $k$-core cover, with $k \in$ $\{1, \ldots,|N|\}$, of a TU-game with player set $N$ as the set of all efficient payoff vectors for which the total joint payoff to any coalition $S$ of size at most $k$ is bounded from above by the value of $S$ in the corresponding dual game, and from below by the value of $S$ in the corresponding minimal rights game. It is shown that the 1-core cover coincides with the core cover, that each $k$-core cover is a core catcher and, interestingly, that the core is a $k$-core cover with ${ }^{1} k=\left\lfloor\frac{|N|}{2}\right\rfloor$.

Defining a game to be $k$-compromise admissible if the $k$-core cover is nonempty, and $k$ compromise stable if it is balanced and the $k$-core cover and core coincide, this paper characterizes $k$-compromise admissible games and $k$-compromise stable games by means of conditions on specific minimal balanced families in both the dual game and the minimal rights game. Finally, we show that assignment games (Shapley and Shubik, 1972) are a specific case of 2compromise stable games.

The paper is structured as follows. Section 2 presents basic definitions and notations regarding TU-games and balanced families. Section 3 introduces and analyzes minimal right games and the $k$-core cover, while Section 4 characterizes $k$-compromise admissible games and $k$-compromise stable games. Section 5 shows that assignment games are 2-compromise stable.

\section{Preliminaries}

A transferable utility game (TU-game) is an ordered pair $(N, v)$ where $N$ is a finite set of players and $v: 2^{N} \rightarrow \mathbb{R}$ satisfies $v(\varnothing)=0$. In general, $v(S)$ represents the value of coalition $S$, that is, the joint payoff that can be obtained by this coalition when its members decide to cooperate. Let $G^{N}$ be the set of all TU-games with player set $N$. Given $S \subseteq N$, let $|S|$ be the number of players in $S$.

The main focus within a cooperative setting is on how to share the total joint payoff obtained when all players decide to cooperate. Given a TU-game $v \in G^{N}$, the core of $v, \mathcal{C}$ ore $(v)$, is defined as the set of efficient allocations (for which exactly $v(N)$ is allocated) that are stable, in the sense

\footnotetext{
${ }^{1}$ For each $r \in \mathbb{R},\lfloor r\rfloor$ denotes the largest integer below or equal to $r$.
} 
that no coalition has an incentive to deviate. Formally,

$$
\operatorname{Core}(v)=\left\{x \in \mathbb{R}^{N}: \sum_{i \in N} x_{i}=v(N), \sum_{i \in S} x_{i} \geq v(S) \text { for all } S \subseteq N\right\} .
$$

It is well known that the core of a game may be empty. In Tijs and Lipperts (1982), the core cover is introduced as a core catcher. The core cover is the set of efficient allocations in which every player gets an amount no lower than his minimal right and no higher than his utopia value. Given a game $v \in G^{N}$ and a player $i \in N$, the utopia value of player $i, M_{i}(v)$, is defined by

$$
M_{i}(v)=v(N)-v(N \backslash\{i\})
$$

and the minimal right of player $i, m_{i}(v)$, is defined by

$$
m_{i}(v)=\max _{S \subseteq N: S \ni i}\left\{v(S)-\sum_{j \in S \backslash\{i\}} M_{j}(v)\right\} .
$$

The core cover of $v \in G^{N}, \mathcal{C C}(v)$, is defined by

$$
\mathcal{C C}(v)=\left\{x \in \mathbb{R}^{N}: \sum_{i \in N} x_{i}=v(N) \text { and } m(v) \leq x \leq M(v)\right\} .
$$

A game $v \in G^{N}$ is compromise admissible if $\mathcal{C C}(v)$ is nonempty. Formally, if

$$
m(v) \leq M(v) \text { and } \quad \sum_{i \in N} m_{i}(v) \leq v(N) \leq \sum_{i \in N} M_{i}(v)
$$

A compromise admissible game is compromise stable if the core cover coincides with the core.

Theorem 2.1 (Tijs and Lipperts (1982)). Let $v \in G^{N}$ be compromise admissible. Then,

(i) $\operatorname{Core}(v) \subseteq \mathcal{C C}(v)$.

(ii) If $|N|=3$, then, $\operatorname{Core}(v)=\mathcal{C C}(v)$.

Quant et al (2005) characterize the class of compromise stable games.

Theorem 2.2 (Quant et al (2005)). Let $v \in G^{N}$ be compromise admissible. Then, Core $(v)=\mathcal{C C}(v)$ if, and only if, for every $S \subseteq N$,

$$
v(S) \leq \max \left\{\sum_{i \in S} m_{i}(v), v(N)-\sum_{i \in N \backslash S} M_{i}(v)\right\} .
$$

Let $\varnothing \neq S \subseteq N$. A family $\mathcal{B}$ of nonempty subcoalitions of $S$ is called balanced on $S$ if there are positive weights $\delta=\left\{\delta_{T}\right\}_{T \in \mathcal{B}}, \delta_{T}>0$ for all $T \in \mathcal{B}$, such that $\sum_{T \in \mathcal{B}} \delta_{T} e^{T}=e^{S}$ or, equivalently, $\sum_{T \in \mathcal{B}: T \ni i} \delta_{T}=1$ for all $i \in S$. Here, $e^{S} \in \mathbb{R}^{N}$ is the characteristic vector of $S$ and is defined 
by $e_{i}^{S}=1$ if $i \in S$ and $e_{i}^{S}=0$ if $i \notin S$. Given a balanced family $\mathcal{B}$, we denote by $\Delta(\mathcal{B})$ the set of positive weights satisfying the balancedness condition. For each $k=1, \ldots,|N|$, we denote by $\mathcal{F}_{k}(S)$ the collection of balanced families on $S$ such that for all $\mathcal{B} \in \mathcal{F}_{k}(S)$ and $R \in \mathcal{B},|R| \leq k$. A balanced family $\mathcal{B} \in \mathcal{F}_{k}(S)$ is minimal if $\mathcal{B}^{\prime} \subseteq \mathcal{B}$ and $\mathcal{B}^{\prime} \in \mathcal{F}_{k}(S)$ implies $\mathcal{B}^{\prime}=\mathcal{B}$. We denote by $\mathcal{F}_{k}^{m}(S)$ the collection of minimal balanced families on $S$. It is well known that a minimal balanced family has a unique vector of balanced weights. Given $\mathcal{B} \in \mathcal{F}_{k}^{m}(S)$, we denote by $\left\{\gamma_{T}^{\mathcal{B}}\right\}_{T \in \mathcal{B}}$ the corresponding vector of balanced weights. It is also known that if $\mathcal{B} \in \mathcal{F}_{k}(S) \backslash \mathcal{F}_{k}^{m}(S)$ and $\delta \in \Delta(\mathcal{B})$, then, there exist $\mathcal{B}_{1}, \ldots, \mathcal{B}_{r} \in \mathcal{F}_{k}^{m}(S)$ with $r \geq 2$ and $t_{1}, \ldots, t_{r} \in(0,1)$ with $\sum_{l=1}^{r} t_{l}=1$ such that $\mathcal{B}=\cup_{l=1}^{r} \mathcal{B}_{l}$ and $\delta_{R}=\sum_{l \in\{1, \ldots, r\}: \mathcal{B}_{l} \ni R} t_{l} \gamma_{R}^{\mathcal{B}_{l}}$.

A game $v \in G^{N}$ is called balanced if for all balanced families $\mathcal{B} \in \mathcal{F}_{|N|}(N)$ and all $\left\{\delta_{S}\right\}_{S \in \mathcal{B}} \in$ $\Delta(\mathcal{B}), \sum_{S \in \mathcal{B}} \delta_{S} v(S) \leq v(N)$. Bondareva (1963) and Shapley (1967) established that a game $v \in$ $G^{N}$ has a nonempty core if, and only if, it is balanced. In fact, they show that a game has a nonempty core if, and only if, all balancedness inequalities are satisfied for minimal balanced families on $N$.

\section{A family of core catchers}

\subsection{Utopia and minimal rights games}

In this subsection we introduce the notions of the utopia and minimal rights games associated to a TU-game.

Definition 3.1. Let $v \in G^{N}$. The dual or utopia game, $v_{D}$, is defined by

$$
v_{D}(S)=v(N)-v(N \backslash S) \quad \text { for all } S \subseteq N
$$

The minimal rights game, $v_{m}$, is defined by

$$
v_{m}(S)=\max _{T \subseteq N: T \supseteq S}\left\{v(T)-\max _{\mathcal{B} \in \mathcal{F}_{|S|}^{m}(T \backslash S)} \sum_{R \in \mathcal{B}} \gamma_{R}^{\mathcal{B}} v_{D}(R)\right\} \quad \text { for all } S \subseteq N .
$$

Note that, for each $S \subseteq N, v_{D}(S)$ reflects the marginal contribution of coalition $S$ to the grand coalition $N$. Therefore, if coalition $S$ asks for a higher share of $v(N)$ than $v_{D}(S)$, it will be profitable for coalition $N \backslash S$ to avoid cooperation with the players of $S$. Accordingly, $v_{D}(S)$ can be interpreted as a utopia value for coalition $S$. Once the values of the utopia game are known to all players, the question is how to compute the minimal rights game. Following the general idea of the minimal rights of a player, we first have to consider what is left from the value of coalition $T$, with $S \subseteq T$, once the players in $T \backslash S$ are paid using the utopia game; secondly, coalition $S$ will maximize its benefit over all potential partners $T \backslash S$, with $S \subseteq T$. Clearly, the difficulty encountered when defining the value of a coalition $S$ in the minimal rights game is how to determine the amount that $S$ should concede to the players of $T \backslash S$, with $S \subseteq T$, according to the utopia game. Using a common pessimistic approach, we consider that this 
quantity is the maximum expected utopia value that coalition $T \backslash S$ can achieve when only coalitions of size at most $|S|$ are considered. Note that, by only considering balanced families of cardinality at most $|S|$, we are generalizing the concept of minimal right of a player. In fact, given a player $i$ and a coalition $T$ with $i \in T$, player $i$ concedes $\sum_{j \in T \backslash\{i\}} M_{j}(v)$ to the players in $T \backslash\{i\}$, where $\{\{j\}: j \in T \backslash\{i\}\}$ is the only minimal balanced family in $\mathcal{F}_{|\{i\}|}^{m}(T \backslash\{i\})$. To conclude, note that if $|T \backslash S|<|S|$, then, $\mathcal{F}_{|T \backslash S|}^{m}(T \backslash S)=\mathcal{F}_{|S|}^{m}(T \backslash S)$ since all coalitions in any minimal balanced family in $\mathcal{F}_{|S|}^{m}(T \backslash S)$ are contained in $T \backslash S$.

The following result states that the maximum expected utopia value for a coalition $T$ over all balanced families of elements with cardinality at most $k$ and over all associated positive weights is achieved in a minimal balanced family. Therefore, the definition of minimal rights game coincides with our informal description.

Proposition 3.2. Let $v \in G^{N}, T \subseteq N$, and $k \in\{1, \ldots,|T|\}$. Then,

$$
\max _{\mathcal{B} \in \mathcal{F}_{k}(T)} \max _{\delta \in \Delta(\mathcal{B})} \sum_{R \in \mathcal{B}} \delta_{R} v_{D}(R)=\max _{\mathcal{B} \in \mathcal{F}_{k}^{m}(T)} \sum_{R \in \mathcal{B}} \gamma_{R}^{\mathcal{B}} v_{D}(R)
$$

Proof. Let $\mathcal{B} \in \mathcal{F}_{k}(T) \backslash \mathcal{F}_{k}^{m}(T)$ and let $\left\{\delta_{R}\right\}_{R \in \mathcal{B}} \in \Delta(\mathcal{B})$. Let $\mathcal{B}_{1}, \ldots, \mathcal{B}_{r} \in \mathcal{F}_{k}^{m}(T)$ and $t_{1}, \ldots, t_{r} \in$ $(0,1)$ with $\sum_{l=1}^{r} t_{l}=1$ satisfy $\mathcal{B}=\cup_{l=1}^{r} \mathcal{B}_{l}$ and $\delta_{R}=\sum_{l \in\{1, \ldots, r\}: \mathcal{B}_{l} \ni R} t_{l} \gamma_{R}^{\mathcal{B}_{l}}$. Then,

$$
\begin{aligned}
\sum_{R \in \mathcal{B}} \delta_{R} v_{D}(R) & =\sum_{R \in \mathcal{B}}\left(\sum_{\substack{l \in\left\{1, \ldots, r \\
\mathcal{B}_{l}, r R\right.}} t_{l} \gamma_{R}^{\mathcal{B}_{l}}\right) v_{D}(R)=\sum_{l=1}^{r} t_{l} \sum_{R \in \mathcal{B}_{l}} \gamma_{R}^{\mathcal{B}_{l}} v_{D}(R) \\
& \leq \sum_{l=1}^{r} t_{l} \max _{\mathcal{B} \in \mathcal{F}_{k}^{m}(T)}\left\{\sum_{R \in \mathcal{B}} \gamma_{R}^{\mathcal{B}} v_{D}(R)\right\}=\max _{\mathcal{B} \in \mathcal{F}_{k}^{m}(T)}\left\{\sum_{R \in \mathcal{B}} \gamma_{R}^{\mathcal{B}} v_{D}(R)\right\}
\end{aligned}
$$

Therefore, $\max _{\mathcal{B} \in \mathcal{F}_{k}(T)} \max _{\delta \in \Delta(\mathcal{B})} \sum_{R \in \mathcal{B}} \delta_{R} v_{D}(R)=\max _{\mathcal{B} \in \mathcal{F}_{k}^{m}(T)} \sum_{R \in \mathcal{B}} \gamma_{R}^{\mathcal{B}} v_{D}(R)$.

Example 3.3. Consider the game $v \in G^{N}$ given in Table 1, where also the values of the utopia and minimal rights games are provided. Next, we illustrate the computation of $v_{m}(\{1\}), v_{m}(\{1,2\})$, and $v_{m}(\{1,2,3\})$.

$$
\begin{aligned}
& v_{m}(\{1\})=\max _{T \subseteq N: T \supseteq\{1\}}\left\{v(T)-\max _{\mathcal{B} \in \mathcal{F}_{1}^{m}(T \backslash S)} \sum_{R \in \mathcal{B}} \gamma_{R}^{\mathcal{B}} v_{D}(R)\right\} \\
& =\max \left\{v(\{1\}), v(\{1,2\})-v_{D}(\{2\}), v(\{1,3\})-v_{D}(\{3\}), v(\{1,4\})-v_{D}(\{4\}),\right. \\
& v(\{1,2,3\})-v_{D}(\{2\})-v_{D}(\{3\}), v(\{1,2,4\})-v_{D}(\{2\})-v_{D}(\{4\}), \\
& \left.v(\{1,3,4\})-v_{D}(\{3\})-v_{D}(\{4\}), v(N)-v_{D}(\{2\})-v_{D}(\{3\})-v_{D}(\{4\})\right\} \\
& =\max \{1,2-2,3-3,5-4,6-2-3,7-2-4,8-3-4,10-2-3-4\}=1 \text {. } \\
& v_{m}(\{1,2\})=\max _{T \subseteq N: T \supseteq\{1,2\}}\left\{v(T)-\max _{\mathcal{B} \in \mathcal{F}_{2}^{m}(T \backslash S)} \sum_{R \in \mathcal{B}} \gamma_{R}^{\mathcal{B}} v_{D}(R)\right\} \\
& =\max \left\{v(\{1,2\}), v(\{1,2,3\})-v_{D}(\{3\}), v(\{1,2,4\})-v_{D}(\{4\}),\right. \\
& \left.v(N)-\max \left\{v_{D}(\{3\})+v_{D}(\{4\}), v_{D}(\{3,4\})\right\}\right\} \\
& =\max \{2,6-3,7-4,10-\max \{3+4,8\}\}=3 \text {. }
\end{aligned}
$$




$$
\begin{aligned}
v_{m}(\{1,2,3\}) & =\max _{T \subseteq N: T \supseteq\{1,2,3\}}\left\{v(T)-\max _{\mathcal{B} \in \mathcal{F}_{3}^{m}(T \backslash S)} \sum_{R \in \mathcal{B}} \gamma_{R}^{\mathcal{B}} v_{D}(R)\right\} \\
& =\max \left\{v(\{1,2,3\}), v(N)-v_{D}(\{4\})\right\} \\
& =\max \{6,10-4\}=6 .
\end{aligned}
$$

\begin{tabular}{|c|c|c|c|c|c|c|c|c|c|c|}
\hline$S$ & $\{1\}$ & $\{2\}$ & $\{3\}$ & $\{4\}$ & $\{1,2\}$ & $\{1,3\}$ & $\{1,4\}$ & $\{2,3\}$ & $\{2,4\}$ & $\{3,4\}$ \\
\hline$v(S)$ & 1 & 1 & 2 & 3 & 2 & 3 & 5 & 3 & 4 & 5 \\
\hline$v_{D}(S)$ & 2 & 2 & 3 & 4 & 5 & 6 & 7 & 5 & 7 & 8 \\
\hline$v_{m}(S)$ & 1 & 1 & 2 & 3 & 3 & 4 & 5 & 4 & 5 & 6 \\
\hline
\end{tabular}

\begin{tabular}{|c|c|c|c|c|c|}
\hline$S$ & $\{1,2,3\}$ & $\{1,2,4\}$ & $\{1,3,4\}$ & $\{2,3,4\}$ & $\{1,2,3,4\}$ \\
\hline$v(S)$ & 6 & 7 & 8 & 8 & 10 \\
\hline$v_{D}(S)$ & 7 & 8 & 9 & 9 & 10 \\
\hline$v_{m}(S)$ & 6 & 7 & 8 & 8 & 10 \\
\hline
\end{tabular}

Table 1: Utopia and minimal rights games in Example 3.3.

The following proposition gives some straightforward implications of the definitions of utopia and minimal rights games. In fact, statement (a) implies that utopia and minimal rights games generalize the utopia values and minimal rights of players. We recall that a game $v \in G^{N}$ is monotone if $v(S) \leq v(T)$ for every $S \subseteq T \subseteq N$. A game $v \in G^{N}$ is convex (see Shapley, 1971) if $v(S \cup\{i\})-v(S) \leq v(T \cup\{i\})-v(T)$ for every $i \in N$ and $S \subseteq T \subseteq N \backslash\{i\}$.

Proposition 3.4. Let $v \in G^{N}$. Then,

a) $v_{D}(\{i\})=M_{i}(v)$ and $v_{m}(\{i\})=m_{i}(v)$ for every $i \in N$.

b) $v_{D}(N)=v_{m}(N)=v(N)$ and $v_{m}(N \backslash\{i\})=v(N \backslash\{i\})$ for all $i \in N$.

c) $v_{m} \geq v$.

d) If $v \in G^{N}$ is monotone, then, $v_{D}$ is non-negative and monotone.

e) If $v \in G^{N}$ is convex, then, $v_{m}=v$.

Proof. The first three items are straightforward.

d) Let $v$ be monotone. Then, $v_{D}(S)=v(N)-v(N \backslash S) \geq 0$ for all $S \subseteq N$. If $S \subseteq T$, then, $N \backslash S \supseteq N \backslash T$, and $v_{D}(S)=v(N)-v(N \backslash S) \leq v(N)-v(N \backslash T)=v_{D}(T)$; therefore, $v_{D}$ is monotone.

e) Let $v$ be convex. First, we show that for all $S \subseteq R \subseteq N$,

$$
v(S) \geq v(R)-\sum_{i \in R \backslash S} v_{D}(\{i\})
$$

or, equivalently, that $v(R)-v(S) \leq \sum_{i \in R \backslash S} v_{D}(\{i\})$. Let $R \backslash S=\left\{i_{1}, \ldots, i_{r}\right\}$. Then,

$$
v(R)-v(S)=v(S \cup(R \backslash S))-v(S)
$$




$$
\begin{aligned}
& =v\left(S \cup\left\{i_{1}, \ldots, i_{r}\right\}\right)-v\left(S \cup\left\{i_{1}, \ldots, i_{r-1}\right\}\right) \\
& +v\left(S \cup\left\{i_{1}, \ldots, i_{r-1}\right\}\right)-v\left(S \cup\left\{i_{1}, \ldots, i_{r-2}\right\}\right)+\ldots \\
& +v\left(S \cup\left\{i_{1}\right\}\right)-v(S) \\
& \leq v(N)-v\left(N \backslash\left\{i_{r}\right\}\right)+v(N)-v\left(N \backslash\left\{i_{r-1}\right\}\right)+\ldots \\
& +v(N)-v\left(N \backslash\left\{i_{1}\right\}\right) \\
& =\sum_{i \in R \backslash S} v_{D}(\{i\})
\end{aligned}
$$

where the inequality follows from convexity of $v$.

Next, we show that for all $S \subseteq R \subseteq N$,

$$
\max _{\mathcal{B} \in \mathcal{F}_{|S|}^{m}(R \backslash S)} \sum_{U \in \mathcal{B}} \gamma_{U}^{\mathcal{B}} v_{D}(U)=\sum_{i \in R \backslash S} v_{D}(\{i\})
$$

Consider $\mathcal{B} \in \mathcal{F}_{|S|}^{m}(R \backslash S)$. Note that for all $U \in \mathcal{B}, v_{D}(U) \leq \sum_{i \in U} v_{D}(\{i\})$ as a consequence of applying (2) to $R=N$ and $S=N \backslash U$. Then,

$$
\sum_{U \in \mathcal{B}} \gamma_{U}^{\mathcal{B}} v_{D}(U) \leq \sum_{U \in \mathcal{B}} \gamma_{U}^{\mathcal{B}} \sum_{i \in U} v_{D}(\{i\})=\sum_{i \in R \backslash S} v_{D}(\{i\})
$$

Clearly, with $\mathcal{B}=\{\{i\}: i \in R \backslash S\} \in \mathcal{F}_{|S|}^{m}(R \backslash S)$ and $\gamma_{U}^{\mathcal{B}}=1$ for every $U \in \mathcal{B}$, we have $\sum_{U \in \mathcal{B}} \gamma_{U}^{\mathcal{B}} v_{D}(U)=\sum_{i \in R \backslash S} v_{D}(\{i\})$ and (3) is proved.

Then,

$$
\begin{aligned}
v_{m}(S) & =\max _{T \subseteq N: T \supseteq S}\left\{v(T)-\max _{\mathcal{B} \in \mathcal{F}_{|S|}^{m}(T \backslash S)} \sum_{R \in \mathcal{B}} \gamma_{R}^{\mathcal{B}} v_{D}(R)\right\} \\
& =\max _{T \subseteq N: T \supseteq S}\left\{v(T)-\sum_{i \in R \backslash S} v_{D}(\{i\})\right\} \\
& =v(S),
\end{aligned}
$$

where the last equality follows from (2).

\section{$3.2 k$-core covers}

This subsection introduces the $k$-core cover of a TU-game $v \in G^{N}$, where $k$ is a natural number between 0 and $|N|$. The $k$-core cover is the set of efficient allocations in which every coalition of size less than or equal to $k$ gets an amount no lower than its value of the minimal rights game and no higher than its value of the utopia game. Formally, we have 
Definition 3.5. Let $v \in G^{N}$ and $k \in\{0,1,2, \ldots,|N|\}$. The $k$-core cover of $v, \mathcal{C C}^{k}(v)$, is defined by

$$
\mathcal{C C}^{k}(v)=\left\{x \in \mathbb{R}^{N:}: \begin{array}{l}
\sum_{i \in N} x_{i}=v(N) \text { and } \\
v_{m}(S) \leq \sum_{i \in S} x_{i} \leq v_{D}(S) \text { for every } S \subseteq N \text { with }|S| \leq k
\end{array}\right\} .
$$

Note that $\mathcal{C C}^{0}(v)=\left\{x \in \mathbb{R}^{N}: \sum_{i \in N} x_{i}=v(N)\right\}, \mathcal{C C}^{1}(v)=\mathcal{C C}(v)$, and $\mathcal{C C}^{|N|-1}(v)=$ $\mathcal{C C}^{|N|}(v)$

Remark 3.1. Equivalently, the $k$-core cover can be recursively defined as follows.

1. $\mathcal{C C}^{0}(v)=\left\{x \in \mathbb{R}^{N}: \sum_{i \in N} x_{i}=v(N)\right\}$.

2. For $k=1, \ldots,|N|$,

$$
\mathcal{C C}^{k}(v)=\left\{x \in \mathcal{C C}^{k-1}(v): v_{m}(S) \leq \sum_{i \in S} x_{i} \leq v_{D}(S) \text { for every } S \subseteq N \text { with }|S|=k\right\} .
$$

The following result states that any $k$-core cover is a core catcher.

Theorem 3.6. Let $v \in G^{N}$. Then,

$$
\varnothing \neq \mathcal{C C}^{0}(v) \supseteq \mathcal{C C}^{1}(v) \supseteq \mathcal{C C}^{2}(v) \supseteq \ldots \supseteq \mathcal{C C}^{|N|-1}(v)=\mathcal{C C}^{|N|}(v)=\operatorname{Core}(v) .
$$

Proof. Clearly, we only have to show the last equality.

First, we show " $\subseteq$ ". For this, let $x \in \mathcal{C C}^{|N|}(v)$. We show that $x \in \mathcal{C}$ ore $(v)$. Note that $\sum_{i \in N} x_{i}=v(N)$ and for every $S \subseteq N, \sum_{i \in S} x_{i} \geq v_{m}(S) \geq v(S)$ where the last inequality follows from Proposition $3.4 \mathrm{c})$. Therefore, $x \in \mathcal{C}$ ore $(v)$.

Second, we show " $\supseteq$ ". Let $x \in \mathcal{C}$ ore $(v)$. We show that $x \in \mathcal{C C}^{|N|}(v)$. Note that $\sum_{i \in N} x_{i}=v(N)$, hence, we only have to show that $v_{m}(S) \leq \sum_{i \in S} x_{i} \leq v_{D}(S)$ for every $S \subseteq N$. Let $S \subseteq N$. To see that $\sum_{i \in S} x_{i} \leq v_{D}(S)$, note that

$$
\sum_{i \in S} x_{i}=\sum_{i \in N} x_{i}-\sum_{i \in N \backslash S} x_{i}=v(N)-\sum_{i \in N \backslash S} x_{i} \leq v(N)-v(N \backslash S)=v_{D}(S) .
$$

To show that $\sum_{i \in S} x_{i} \geq v_{m}(S)$, let $T \subseteq N$ with $S \subseteq T$. Then, for every $\mathcal{B} \in \mathcal{F}_{|S|}^{m}(T \backslash S)$, we have that

$$
v(T) \leq \sum_{i \in T} x_{i}=\sum_{i \in S} x_{i}+\sum_{i \in T \backslash S} x_{i}=\sum_{i \in S} x_{i}+\sum_{R \in \mathcal{B}} \gamma_{R}^{\mathcal{B}} \sum_{i \in R} x_{i} \leq \sum_{i \in S} x_{i}+\sum_{R \in \mathcal{B}} \gamma_{R}^{\mathcal{B}} v_{D}(R)
$$

where the second inequality follows from Equation (4). Hence,

$$
\sum_{i \in S} x_{i} \geq v(T)-\sum_{R \in \mathcal{B}} \gamma_{R}^{\mathcal{B}} v_{D}(R) \text { for every } \mathcal{B} \in \mathcal{F}_{|S|}^{m}(T \backslash S)
$$


Consequently, $\sum_{i \in S} x_{i} \geq \max _{T \subseteq N: T \supseteq S}\left\{v(T)-\max _{\mathcal{B} \in \mathcal{F}_{|S|}^{m}(T \backslash S)} \sum_{R \in \mathcal{B}} \gamma_{R}^{\mathcal{B}} v_{D}(R)\right\}=v_{m}(S)$.

Using Theorem 3.6, it can be shown that the core of a game coincides with the core of its minimal rights game.

Theorem 3.7. Let $v \in G^{N}$ and $v_{m}$ its minimal rights game. Then, $\operatorname{Core}(v)=\operatorname{Core}\left(v_{m}\right)$.

Proof. Note that $\operatorname{Core}\left(v_{m}\right) \subseteq \mathcal{C}$ ore $(v)$ since for all $S \subseteq N, v_{m}(S) \geq v(S)$ while $v_{m}(N)=v(N)$. Then, we only have to show that $\operatorname{Core}\left(v_{m}\right) \supseteq \operatorname{Core}(v)$. Let $x \in \operatorname{Core}(v)$. Then, $\operatorname{Core}(v)=$ $\mathcal{C C}^{|N|}(v)$ by Theorem 3.6 and $\sum_{i \in S} x_{i} \geq v_{m}(S)$ for all $S \subseteq N$. Consequently, $x \in \operatorname{Core}\left(v_{m}\right)$.

Moreover, it turns out that the core of a game with set of players $N$ coincides with the $\left\lfloor\frac{|N|}{2}\right\rfloor$ core cover. From this fact, one can easily derive the coincidence of the core and the 1-core cover for arbitrary 3-player games (cf. Theorem 2.1 (ii)).

Theorem 3.8. Let $v \in G^{N}$. Then, $\operatorname{Core}(v)=\mathcal{C C}^{\left\lfloor\frac{|N|}{2}\right\rfloor}(v)$.

Proof. Using Theorem 3.6, it is sufficient to show that $\mathcal{C o r e}(v) \supseteq \mathcal{C C}^{\left\lfloor\frac{|N|}{2}\right\rfloor}(v)$. Let $x \in \mathcal{C C}^{\left\lfloor\frac{|N|}{2}\right\rfloor}(v)$. Clearly, $\sum_{i \in N} x_{i}=v(N)$. Let $S \subseteq N$ with $|S| \leq\left\lfloor\frac{|N|}{2}\right\rfloor$. Then,

$$
\sum_{i \in S} x_{i} \geq v_{m}(S) \geq v(S)
$$

where the first inequality is a direct consequence of the definition of $\left\lfloor\frac{|N|}{2}\right\rfloor$-core cover and the second inequality follows from Proposition 3.4 c). Next, let $S \subseteq N$ with $|S|>\left\lfloor\frac{|N|}{2}\right\rfloor$. Then,

$$
\sum_{i \in S} x_{i}=\sum_{i \in N} x_{i}-\sum_{i \in N \backslash S} x_{i}=v(N)-\sum_{i \in N \backslash S} x_{i} \geq v(N)-v_{D}(N \backslash S)=v(S)
$$

where the first inequality is a direct consequence of $x \in \mathcal{C C}^{\left\lfloor\frac{|N|}{2}\right\rfloor}(v)$ and $0<|N \backslash S|<\left\lfloor\frac{|N|}{2}\right\rfloor$. Consequently, $x \in \operatorname{Core}(v)$.

As an immediate consequence of Theorem 3.6 and Theorem 3.8, we have the following result.

Corollary 3.9. Let $v \in G^{N}$. Then, $\mathcal{C C}^{k}(v)=\operatorname{Core}(v)$ for all $k \geq\left\lfloor\frac{\lfloor N \mid}{2}\right\rfloor$.

Since, by Theorem 3.6, the $k$-core cover is contained in the $l$-core cover for every $l<k$, it is useful to define the smallest nonempty $k$-core cover of a game.

Definition 3.10. For $v \in G^{N}$, the least core cover, $\mathcal{L C C}(v)$, is defined by $\operatorname{LCC}(v)=\mathcal{C C}^{k^{*}}(v)$ where $k^{*}=\max \left\{k \in\left\{0,1, \ldots,\left\lfloor\frac{|N|}{2}\right\rfloor\right\}: \mathcal{C C}^{k}(v) \neq \varnothing\right\}$.

Note that the least core cover is a nonempty core catcher. Besides, if $v$ is balanced, then, $\operatorname{LCC}(v)=\operatorname{Core}(v)$. The following example illustrates the least core cover of a game with an empty core. 
Example 3.11. Consider the 6-player game $v \in G^{N}$ where the characteristic function is given by

$$
v(S)= \begin{cases}0 & \text { if }|S|=1, \\ 2 & \text { if }|S|=2, \\ 5 & \text { if }|S|=3, \\ 4 & \text { if }|S|=4, \\ 5 & \text { if }|S|=5, \\ 8 & \text { if }|S|=6 .\end{cases}
$$

First, we show that this game has an empty core. For this, suppose that the core is nonempty and let $x \in \operatorname{Core}(v)$. Then, $x_{1}+x_{2}+x_{3} \geq 5$ and $x_{4}+x_{5}+x_{6} \geq 5$. Adding both inequalities and taking into account that $\sum_{i=1}^{6} x_{i}=v(N)=8$ since $x \in \mathcal{C}$ ore $(v)$, we obtain $8=\sum_{i=1}^{6} x_{i} \geq 10$, establishing a contradiction. According to Theorem $3.8, \mathcal{C C}^{3}(v)=\mathcal{C}$ ore $(v)=\varnothing$. It turns out that the game has a nonempty 2-core cover. We subsequently compute the 1- and 2-core covers.

Note that $v_{D}(\{i\})=v(N)-v(N \backslash\{i\})=3$ for all $i \in N$ and

$$
v_{m}(\{i\})=\max \left\{v(\{i\}), \max \left\{v(S)-\sum_{j \in S \backslash\{i\}} v_{D}(\{j\}): S \subseteq N, i \in S\right\}\right\}=0
$$

for all $i \in N$. Then, $v_{m}(\{i\}) \leq v_{D}(\{i\})$ for all $i \in N$ and $\sum_{i \in N} v_{m}(\{i\}) \leq v(N) \leq \sum_{i \in N} v_{D}(\{i\})$. Therefore, $\mathcal{C C}^{1}(v) \neq \varnothing$ and it is given $b y^{2}$

$$
\mathcal{C C}^{1}(v)=\operatorname{con}\left(\left\{3 e^{\{i\}}+3 e^{\{j\}}+2 e^{\{k\}}: i, j, k \in N,|\{i, j, k\}|=3\right\}\right) .
$$

Next, we compute the 2-core cover. Note that $v_{D}(\{i, j\})=v(N)-v(N \backslash\{i, j\})=8-4=4$ for all $i, j \in N$ with $i \neq j$. Moreover, for all $i, j \in N$ with $i \neq j$ and all $S \subseteq N$ with $i, j \in S$ and $|S| \geq 4$, we have that $v(S)-\max _{\mathcal{B} \in \mathcal{F}_{2}^{m}(S \backslash\{i, j\})} \sum_{R \in \mathcal{B}} \gamma_{R}^{\mathcal{B}} v_{D}(R) \leq 0$. Thus, for all $i, j \in N$ with $i \neq j$,

$$
v_{m}(\{i, j\})=\max \left\{v(\{i, j\}), \max \left\{v(\{i, j, k\})-v_{D}(\{k\}): k \in N \backslash\{i, j\}\right\}\right\}=2 .
$$

Then, for all $i, j \in N$ with $i \neq j, v_{m}(\{i, j\}) \leq v_{D}(\{i, j\})$ and

$$
\mathcal{C C}^{2}(v)=\operatorname{con}\left(\left\{e^{N}+2 e^{\{i\}}: i \in N\right\}\right)=\mathcal{L C C}(v)
$$

\section{$4 k$-compromise admissibility and $k$-compromise stability}

\section{1 k-compromise admissible games}

Definition 4.1. For $k \in\{0,1, \ldots,|N|\}$, a game $v \in G^{N}$ is $k$-compromise admissible if $\mathcal{C C}^{k}(v)$ is nonempty.

\footnotetext{
${ }^{2}$ Given a finite set $A \subseteq \mathbb{R}^{N}, \operatorname{con}(A)$ denotes the convex hull of $A$.
} 
Note that any game is 0 -compromise admissible. Before characterizing $k$-compromise admissibility, we introduce the concepts of $k$-core and $k$-anti core.

Definition 4.2. Let $v \in G^{N}$ and $k \in\{1, \ldots,|N|\}$. The $k$-core of $v, \operatorname{Core}^{k}(v)$, is the set of efficient allocations that are stable for coalitions of size smaller than or equal to $k$. Formally,

$$
\operatorname{Core}^{k}(v)=\left\{x \in \mathbb{R}^{N}: \sum_{i \in N} x_{i}=v(N), \sum_{i \in S} x_{i} \geq v(S) \text { for all } S \subseteq N \text { with }|S| \leq k\right\} .
$$

Similarly, the $k$-anti core of $v_{D}, \mathcal{A C o r e}{ }^{k}\left(v_{D}\right)$, is defined by

$$
\mathcal{A C o r e}^{k}\left(v_{D}\right)=\left\{x \in \mathbb{R}^{N}: \sum_{i \in N} x_{i}=v_{D}(N), \sum_{i \in S} x_{i} \leq v_{D}(S) \text { for all } S \subseteq N \text { with }|S| \leq k\right\} \text {. }
$$

The following result follows directly from the definitions of $k$-core and $k$-anti core.

Proposition 4.3. Let $v \in G^{N}$. Then,

$$
\begin{aligned}
& \operatorname{Core}^{1}(v) \supseteq \operatorname{Core}^{2}(v) \supseteq \ldots \supseteq \mathcal{C o r e}^{|N|}(v)=\mathcal{C} \text { ore }(v) \text { and } \\
& \mathcal{A C o r e}^{1}\left(v_{D}\right) \supseteq \mathcal{A C o r e}^{2}\left(v_{D}\right) \supseteq \ldots \supseteq \mathcal{A C o r e} e^{|N|}\left(v_{D}\right)=\mathcal{C} \text { ore }(v) \text {. }
\end{aligned}
$$

As an immediate consequence, we have

Theorem 4.4. Let $v \in G^{N}$ and $k \in\{1, \ldots,|N|\}$. Then, $\mathcal{C C}^{k}(v)=\operatorname{Core}^{k}\left(v_{m}\right) \cap \mathcal{A C o r e}^{k}\left(v_{D}\right)$ and $\operatorname{Core}(v)=\operatorname{Core}^{\left\lfloor\frac{|N|}{2}\right\rfloor}\left(v_{m}\right) \cap \mathcal{A C o r e}^{\left\lfloor\frac{|N|}{2}\right\rfloor}\left(v_{D}\right)$.

Next, we introduce the concepts of $k$-balanced games and $k$-dual balanced games and show that $k$-balancedness ( $k$-dual balancedness) is a sufficient and necessary condition for non-emptiness of the $k$-core ( $k$-anti core).

Definition 4.5. Let $v \in G^{N}$ and $k \in\{1, \ldots,|N|\}$.

- $v$ is $k$-balanced if for all balanced families $\mathcal{B} \in \mathcal{F}_{k}(N)$ and all $\left\{\delta_{S}\right\}_{S \in \mathcal{B}} \in \Delta(\mathcal{B})$,

$$
\sum_{S \in \mathcal{B}} \delta_{S} v(S) \leq v(N)
$$

- $v$ is $k$-dual balanced if for all balanced families $\mathcal{B} \in \mathcal{F}_{k}(N)$ and all $\left\{\delta_{S}\right\}_{S \in \mathcal{B}} \in \Delta(\mathcal{B})$,

$$
\sum_{S \in \mathcal{B}} \delta_{S} v(S) \geq v(N)
$$

Just like for balanced games, one defines $k$-minimal balanced and $k$-minimal dual balanced games based on $k$-minimal balanced families.

The following theorem extends the characterization of Bondareva-Shapley of nonemptiness of the core (Bondareva, 1963; Shapley, 1967) to the nonemptiness of the $k$-core and the $k$-anti core. The proof follows the same lines as the proof in Shapley (1967) and the proof of Theorem 4.8 below and is, therefore, omitted. 
Theorem 4.6. Let $v \in G^{N}$ and $k \in\{1, \ldots,|N|\}$. Then,

a) $\operatorname{Core}^{k}(v) \neq \varnothing$ if, and only if, $v$ is minimally $k$-balanced.

b) $\mathcal{A C o r e}{ }^{k}\left(v_{D}\right) \neq \varnothing$ if, and only if, $v_{D}$ is minimally $k$-dual balanced.

Example 4.7. Consider the 6-player game $v \in G^{N}$ where the characteristic function is given by

$$
v(S)= \begin{cases}0 & \text { if }|S|=1, \\ 3 & \text { if }|S|=2 \text { and } 1 \in S, \\ 2 & \text { if }|S|=2,1 \notin S, \text { and } 2 \in S, \\ 1 & \text { if }|S|=2,1 \notin S, \text { and } 2 \notin S, \\ 0 & \text { if }|S|=3, \\ 4.75 & \text { if }|S|=4 \text { and } 1,2 \in S, \\ 3.75 & \text { if }|S|=4,1 \in S, \text { and } 2 \notin S, \\ 2.75 & \text { if }|S|=4 \text { and } 1 \notin S, \\ 3.25 & \text { if }|S|=5, \\ 6.25 & \text { if } S=N .\end{cases}
$$

It turns out that $\operatorname{Core}^{2}\left(v_{m}\right) \neq \varnothing, \mathcal{A C o r e}^{2}\left(v_{D}\right) \neq \varnothing$, and $\mathcal{C C}^{2}(v)=\operatorname{Core}^{2}\left(v_{m}\right) \cap \mathcal{A C o r e}^{2}\left(v_{D}\right)=\varnothing$. To see this we give the values of the minimal rights game and the utopia game for coalitions of cardinality at most 2. We have that $v_{m}(S)=v(S)$ for every $S \subseteq N$ with $|S| \leq 2$ and

$$
v_{D}(S)= \begin{cases}3 & \text { if }|S|=1, \\ 3.5 & \text { if }|S|=2 \text { and } 1 \in S, \\ 2.5 & \text { if }|S|=2,1 \notin S, \text { and } 2 \in S, \\ 1.5 & \text { if }|S|=2 \text { and } 1,2 \notin S .\end{cases}
$$

Note that $(2.5,1.5,0.5,0.5,0.5,0.75) \in \operatorname{Core}^{2}\left(v_{m}\right)$ and $(2.75,0.75,0.75,0.75,0.75,0.5) \in \mathcal{A C o r e}^{2}\left(v_{D}\right)$. However, $\mathcal{C C}^{2}(v)=\operatorname{Core}^{2}\left(v_{m}\right) \cap \mathcal{A C o r e}^{2}\left(v_{D}\right)=\varnothing$. To see this, suppose that the 2-core cover is nonempty and let $x \in \mathcal{C C}^{2}(v)$. Then, $x_{1}+x_{3} \geq 3, x_{1}+x_{4} \geq 3, x_{2}+x_{5} \geq 2, x_{2}+x_{6} \geq 2$ and $x_{1}+x_{2} \leq 3.5$. Adding the first four inequalities and subtracting the last one, and taking into account that $\sum_{i=1}^{6} x_{i}=v(N)=6.25$, we obtain $6.25=\sum_{i=1}^{6} x_{i} \geq 6.5$.

Next, we characterize the class of $k$-compromise admissible games. Note that $k$-compromise admissibility, for $k \geq\left\lfloor\frac{|N|}{2}\right\rfloor$, is equivalent to balancedness by Theorem 3.8 which is equivalent to balancedness of the minimal rights game by Theorem 3.7. Therefore, we restrict our attention to $k$-compromise admissibility for $k \in\left\{1, \ldots,\left\lfloor\frac{\lfloor N \mid}{2}\right\rfloor-1\right\}$. The formal proof of Theorem 4.8 can be found in the Appendix. Before giving the characterization, we introduce some notation. Let $k \in$ $\left\{1, \ldots,\left\lfloor\frac{|N|}{2}\right\rfloor-1\right\}$. We denote by $\mathcal{F}_{k,|N|-k}(N)$ the set of balanced families on $N$ whose elements have cardinality at most $k$, or at least $|N|-k$. We denote by $\mathcal{F}_{k,|N|-k}^{m}(N)$ the corresponding set of minimal balanced families. 
Theorem 4.8. Let $v \in G^{N}$ and $k \in\left\{1, \ldots,\left\lfloor\frac{|N|}{2}\right\rfloor-1\right\}$. Then, $v$ is $k$-compromise admissible if, and only if, the following condition is satisfied:

$$
\sum_{\substack{R \in \mathcal{B} \\ 0<|R| \leq k}} \gamma_{R}^{\mathcal{B}} v_{m}(R)-\sum_{\substack{R \in \mathcal{B} \\|N|-k \leq|R|<|N|}} \gamma_{R}^{\mathcal{B}} v_{D}(N \backslash R) \leq\left(1-\sum_{\substack{R \in \mathcal{B} \\|N|-k \leq|R|<|N|}} \gamma_{R}^{\mathcal{B}}\right) v(N)
$$

for every $\mathcal{B} \in \mathcal{F}_{k,|N|-k}^{m}(N), \mathcal{B} \neq\{N\}$.

Remark 4.1. (i) Observe that Theorem 4.8 generalizes the characterization of 1-compromise stability given in Equation (1). Recall that, by Proposition $3.4 \mathrm{a}), m_{i}(v)=v_{m}(\{i\})$ and $M_{i}(v)=v_{D}(\{i\})$ for every $i \in N$. Note that $\mathcal{F}_{1,|N|-1}^{m}$ consists of the families: $\{\{i\}: i \in N\}$, $\{\{i\}, N \backslash\{i\}\}$ for every $i \in N,\{N \backslash\{i\}: i \in N\}$ and $\{N\}$.

If $\mathcal{B}=\{\{i\}, N \backslash\{i\}\}$ with $i \in N$, then, $\gamma_{\{i\}}^{\mathcal{B}}=\gamma_{N \backslash\{i\}}^{\mathcal{B}}=1$ and the condition in Theorem 4.8 becomes $m_{i}(v)=v_{m}(\{i\}) \leq v_{D}(\{i\})=M_{i}(v)$.

If $\mathcal{B}=\{\{i\}: i \in N\}$, then, $\gamma_{\{i\}}^{\mathcal{B}}=1$ for every $i \in N$ and the condition in Theorem 4.8 becomes $\sum_{i \in N} m_{i}(v)=\sum_{i \in N} v_{m}(\{i\}) \leq v(N)$.

If $\mathcal{B}=\{N \backslash\{i\}: i \in N\}$, then, $\gamma_{N \backslash\{i\}}^{\mathcal{B}}=\frac{1}{|N|-1}$ for every $i \in N$ and the condition in Theorem 4.8 becomes $\sum_{i \in N} M_{i}(v)=\sum_{i \in N} v_{D}(\{i\}) \geq v(N)$.

(ii) It can be easily seen that the conditions a) $v_{m}(S) \leq v_{D}(S)$ for all $S \subseteq N$ such that $|S| \leq k$ and b) $\sum_{S \in \mathcal{B}} \gamma_{S}^{\mathcal{B}} v_{m}(S) \leq v(N) \leq \sum_{S \in \mathcal{B}} \gamma_{S}^{\mathcal{B}} v_{D}(S)$ for all $\mathcal{B} \in \mathcal{F}_{k}^{m}(N)$ are necessary for $k$ compromise admissibility. They are however not sufficient for $k>1$ as we see below.

Note that the game in Example 4.7 satisfies $v_{m}(S) \leq v_{D}(S)$ for all $S \subseteq N$ such that $|S| \leq 2$ and $\sum_{S \in \mathcal{B}} \gamma_{S}^{\mathcal{B}} v_{m}(S) \leq v(N) \leq \sum_{S \in \mathcal{B}} \gamma_{S}^{\mathcal{B}} v_{D}(S)$ for all $\mathcal{B} \in \mathcal{F}_{2}^{m}(N)$. However, $\mathcal{C C}^{2}(v)=\varnothing$. To see this, we take $\mathcal{B}=\{\{1,3\},\{1,4\},\{2,5\},\{2,6\},\{3,4,5,6\}\} \in \mathcal{F}_{2,|N|-2}^{m}$ where $\gamma_{S}^{\mathcal{B}}=\frac{1}{2}$ for every $S \in \mathcal{B}$. Then,

$$
\begin{aligned}
\sum_{\substack{R \in \mathcal{B} \\
0<|R| \leq 2}} \gamma_{R}^{\mathcal{B}} v_{m}(R)-\sum_{\substack{R \in \mathcal{B} \\
|N|-2 \leq|R|<|N|}} \gamma_{R}^{\mathcal{B}} v_{D}(N \backslash R) \\
=\frac{1}{2}\left(v_{m}(\{1,3\})+v_{m}(\{1,4\})+v_{m}(\{2,5\})+v_{m}(\{2,6\})-v_{D}(N \backslash\{3,4,5,6\})\right) \\
=\frac{1}{2}(3+3+2+2-3.5)=\frac{6.5}{2} \\
>\frac{6.25}{2}=\left(1-\frac{1}{2}\right) 6.25=\left(1-\sum_{\substack{R \in \mathcal{B} \\
|N|-2 \leq|R|<|N|}} \gamma_{R}^{\mathcal{B}}\right) v(N) .
\end{aligned}
$$

\section{$4.2 k$-compromise stable games}

Quant et al (2005) introduce and characterize the class of compromise stable games, which are those games that are compromise admissible and for which the core and the core cover coincide. Here, we perform a similar analysis for so called $k$-compromise stable games. 
Definition 4.9. A k-compromise admissible game $v \in G^{N}$ is called $k$-compromise stable if $\mathcal{C C}^{k}(v)=$ $\operatorname{Core}(v)$.

Note that a $k$-compromise stable game is $k$-compromise admissible and that, consequently, the $k$-core cover, which coincides with the core, is nonempty and the game is balanced. The game in Example 3.11 has an empty core and, therefore, it is not $k$-compromise stable for any $k$.

Example 4.10. Reconsider the game $v \in G^{N}$ in Example 3.3. It is readily checked that this game is 1-compromise stable since $\mathcal{C}$ ore $(v)=\mathcal{C C}^{1}(v)=\operatorname{con}(\{(2,2,3,3),(2,2,2,4),(2,1,3,4),(1,2,3,4)\})$.

Note that for each balanced game, the game is $\left\lfloor\frac{|N|}{2}\right\rfloor$-compromise stable. Our main theorem will provide necessary and sufficient conditions for a balanced game to be $k$-compromise stable with $k \in\left\{1, \ldots,\left\lfloor\frac{|N|}{2}\right\rfloor\right\}$. The next lemma provides necessary and sufficient conditions for $\operatorname{Core}^{k}\left(v_{m}\right)=\operatorname{Core}(v)$ and $\mathcal{A C o r e}{ }^{k}\left(v_{D}\right)=\mathcal{C}$ ore $(v)$.

Lemma 4.11. Let $v \in G^{N}$ be a balanced game and $k \in\left\{1, \ldots,\left\lfloor\frac{|N|}{2}\right\rfloor\right\}$. Then,

a) $\operatorname{Core}^{k}\left(v_{m}\right)=\mathcal{C} \operatorname{Core}(v)$ if, and only if, $v(S) \leq \max _{\mathcal{B} \in \mathcal{F}_{k}^{m}(S)} \sum_{R \in \mathcal{B}} \gamma_{R}^{\mathcal{B}} v_{m}(R)$ for all $S \subseteq N$.

b) $\mathcal{A C o r e}^{k}\left(v_{D}\right)=\mathcal{C o r e}(v)$ if, and only if, $v(S) \leq v(N)-\min _{\mathcal{B} \in \mathcal{F}_{k}^{m}(N \backslash S)} \sum_{R \in \mathcal{B}} \gamma_{R}^{\mathcal{B}} v_{D}(R)$ for all $S \subseteq N$.

c) $\operatorname{Core}^{k}\left(v_{m}\right)=\operatorname{Core}(v)=\mathcal{A C o r e}{ }^{k}\left(v_{D}\right)$ if, and only if, $v(S) \leq \min \left\{\max _{\mathcal{B} \in \mathcal{F}_{k}^{m}(S)} \sum_{R \in \mathcal{B}} \gamma_{R}^{\mathcal{B}} v_{m}(R), v(N)-\min _{\mathcal{B} \in \mathcal{F}_{k}^{m}(N \backslash S)} \sum_{R \in \mathcal{B}} \gamma_{R}^{\mathcal{B}} v_{D}(R)\right\}$ for all $S \subseteq N$.

Proof. We prove a) in detail. The proof of b) can be done following similar arguments and c) is an immediate consequence of a) and b) combined. First, we show the "if" part. Let

$$
v(S) \leq \max _{\mathcal{B} \in \mathcal{F}_{k}^{m}(S)} \sum_{R \in \mathcal{B}} \gamma_{R}^{\mathcal{B}} v_{m}(R)
$$

for all $S \subseteq N$. We show that $\operatorname{Core}^{k}\left(v_{m}\right)=\mathcal{C}$ ore $(v)$. By Proposition 4.3 and Theorem 3.7, we know that $\mathcal{C o r e}^{k}\left(v_{m}\right) \supseteq \mathcal{C} \operatorname{Core}\left(v_{m}\right)=\mathcal{C}$ ore $(v)$. Therefore, we only have to prove that $\operatorname{Core}^{k}\left(v_{m}\right) \subseteq$ $\operatorname{Core}(v)$. Let $x \in \operatorname{Core}^{k}\left(v_{m}\right)$. Then, $\sum_{i \in N} x_{i}=v(N)$. Let $S \subseteq N$ be such that $k<|S|$. We show that $\sum_{i \in S} x_{i} \geq v(S)$. Note that $v_{m}(R) \leq \sum_{i \in R} x_{i}$ for every $R \subseteq N$ with $|R| \leq k$ and for all $\mathcal{B} \in \mathcal{F}_{k}^{m}(S)$,

$$
\sum_{i \in S} x_{i}=\sum_{R \in \mathcal{B}} \gamma_{R}^{\mathcal{B}} \sum_{i \in R} x_{i} \geq \sum_{R \in \mathcal{B}} \gamma_{R}^{\mathcal{B}} v_{m}(R) .
$$

Therefore, using (5), $\sum_{i \in S} x_{i} \geq \max _{\mathcal{B} \in \mathcal{F}_{k}^{m}(S)} \sum_{R \in \mathcal{B}} \gamma_{R}^{\mathcal{B}} v_{m}(R) \geq v(S)$.

Next, we show the "only if" part. Let $\operatorname{Core}^{k}\left(v_{m}\right)=\operatorname{Core}(v)$. First, note that $\operatorname{Core}^{k}\left(v_{m}\right)$ is nonempty and can be obtained as the set of optimal solutions of the linear programming problem $\left(P_{1}\right)$ :

$$
\left(P_{1}\right) \quad \min \sum_{i \in N} x_{i}
$$




$$
\begin{aligned}
& \sum_{i \in R} x_{i} \geq v_{m}(R) \text { for every } R \subseteq N,|R| \leq k, \\
& \sum_{i \in N} x_{i} \geq v(N) .
\end{aligned}
$$

Let $S \subseteq N$. If $1 \leq|S| \leq k$, then, clearly,

$$
v(S) \leq v_{m}(S) \leq \max _{\mathcal{B} \in \mathcal{F}_{k}^{m}(S)} \sum_{R \in \mathcal{B}} \gamma_{R}^{\mathcal{B}} v_{m}(R)
$$

where the second inequality follows from the fact that $\{S\} \in \mathcal{F}_{k}^{m}(S)$ with $\gamma_{S}^{\{\mathcal{S}\}}=1$. Take now $|S| \geq k+1$. Since $\operatorname{Core}^{k}\left(v_{m}\right)=\mathcal{C}$ ore $(v)=\operatorname{Core}\left(v_{m}\right)$, we know that $\sum_{i \in S} x_{i} \geq v_{m}(S)$ for every $x \in \operatorname{Core}^{k}\left(v_{m}\right)$. Therefore, we can now modify problem $\left(P_{1}\right)$ into problem $\left(P_{2}\right)$

$$
\begin{aligned}
& \min \sum_{i \in N} x_{i} \\
& \sum_{i \in R} x_{i} \geq v_{m}(R) \text { for every } R \subseteq N,|R| \leq k, \\
& \sum_{i \in S} x_{i} \geq v_{m}(S) \text { for every } S \subseteq N,|S| \geq k+1, \\
& \sum_{i \in N} x_{i} \geq v(N)
\end{aligned}
$$

where for each $S \subseteq N$ with $|S| \geq k+1$, the inequality constraint $\sum_{i \in S} x_{i} \geq v_{m}(S)$ is redundant. As a consequence, for every $S \subseteq N$ with $|S| \geq k+1$, there exists a non-negative linear combination of the constraint inequalities in $\left(P_{1}\right)$ such that the linear combination makes the inequality $\sum_{i \in S} x_{i} \geq v_{m}(S)$ redundant. That is, there exists $\left\{\delta_{R}\right\}_{R \subseteq S,|R| \leq k}$ with $\delta_{R} \geq 0$ for each $R \subseteq S$ with $|R| \leq k$ and $\sum_{R} \delta_{R}=1$, or equivalently, there is $\mathcal{B} \in \mathcal{F}_{k}(S)$ and $\left\{\delta_{R}\right\}_{R \in \mathcal{B}} \in \Delta(\mathcal{B})$ such that

$$
\sum_{i \in S} x_{i}=\sum_{R \in \mathcal{B}} \delta_{R} \sum_{i \in R} x_{i} \geq \sum_{R \in \mathcal{B}} \delta_{R} v_{m}(R) \geq v_{m}(S)
$$

Then, $v(S) \leq v_{m}(S) \leq \max _{\mathcal{B} \in \mathcal{F}_{k}(S)} \max _{\left\{\delta_{R}\right\}_{R \in \mathcal{B}} \in \Delta(\mathcal{B})} \sum_{R \in \mathcal{B}} \delta_{R} v_{m}(R)=\max _{\mathcal{B} \in \mathcal{F}_{k}^{m}(S)} \sum_{R \in \mathcal{B}} \gamma_{R}^{\mathcal{B}} v_{m}(R)$, where the first inequality is a direct consequence of Proposition $3.4 \mathrm{C}$ ) and the last equality is based on the same type of argument as in the proof of Proposition 3.2.

Note that Lemma $4.11 \mathrm{c}$ ) provides a sufficient condition for $k$-compromise admissibility. The following result provides a full characterization of the class of $k$-compromise stable games.

Theorem 4.12. Let $v \in G^{N}$ be a balanced game and $k \in\left\{1, \ldots,\left\lfloor\frac{|N|}{2}\right\rfloor\right\}$. Then, $v$ is $k$-compromise stable if, and only if, for every $S \subseteq N$,

$$
v(S) \leq \max \left\{\max _{\mathcal{B} \in \mathcal{F}_{k}^{m}(S)} \sum_{R \in \mathcal{B}} \gamma_{R}^{\mathcal{B}} v_{m}(R), v(N)-\min _{\mathcal{B} \in \mathcal{F}_{k}^{m}(N \backslash S)} \sum_{R \in \mathcal{B}} \gamma_{R}^{\mathcal{B}} v_{D}(R)\right\} .
$$

Proof. We start showing the "only if" part. Let $k \in\left\{1, \ldots,\left\lfloor\frac{|N|}{2}\right\rfloor\right\}$ and assume that $\mathcal{C C}^{k}(v)=$ 
$\operatorname{Core}(v)$. We show that (7) is satisfied. Let $S \subseteq N$ be such that $|S| \leq k$. Then,

$$
v(S) \leq v_{m}(S) \leq \max _{\mathcal{B} \in \mathcal{F}_{k}^{m}(S)} \sum_{R \in \mathcal{B}} \gamma_{R}^{\mathcal{B}} v_{m}(R)
$$

where the second inequality follows by considering $\mathcal{B}=\{S\}$ and $\gamma_{S}^{\mathcal{B}}=1$. Therefore, (7) follows.

Let $S \subseteq N$ be such that $|S|>k$. If $v(S) \leq \max _{\mathcal{B} \in \mathcal{F}_{k}^{m}(S)} \sum_{R \in \mathcal{B}} \gamma_{R}^{\mathcal{B}} v_{m}(R)$, then (7) follows. Therefore, assume that

$$
v(S)>\max _{\mathcal{B} \in \mathcal{F}_{k}^{m}(S)} \sum_{R \in \mathcal{B}} \gamma_{R}^{\mathcal{B}} v_{m}(R)
$$

Note that Theorem 3.6 and the assumption $\mathcal{C C}^{k}(v)=\operatorname{Core}(v)$ imply $\mathcal{C C}^{k}(v)=\mathcal{C C}^{l}(v)=\operatorname{Core}(v)$ for all $l>k$. Therefore, the inequalities $\sum_{i \in R} x_{i} \geq v_{m}(R)$, with $|R|>k$, are redundant in the description of $\mathcal{C C}^{l}(v)$ for every $l \geq|R|$ and, in particular, $\sum_{i \in S} x_{i} \geq v_{m}(S)$ is also redundant in the description of $\mathcal{C C}^{l}(v)$ for every $l \geq|S|$. Now, suppose that we can derive $\sum_{i \in S} x_{i} \geq v_{m}(S)$ as a positive linear combination of inequalities of the form $\sum_{i \in R} x_{i} \geq v_{m}(R)$ with $R \subseteq N,|R| \leq k$. Then, there exists $\mathcal{B}_{1} \in \mathcal{F}_{k}^{m}(S)$ such that

$$
\sum_{i \in S} x_{i}=\sum_{R \in \mathcal{B}_{1}} \gamma_{R}^{\mathcal{B}_{1}} \sum_{i \in R} x_{i} \geq \sum_{R \in \mathcal{B}_{1}} \gamma_{R}^{\mathcal{B}_{1}} v_{m}(R) \geq v_{m}(S) \geq v(S)
$$

establishing a contradiction with (8). Therefore, there must exist a linear combination of inequalities of the form $\sum_{i \in R} x_{i} \leq v_{D}(R)$ with $R \subseteq N,|R| \leq k$, that makes $\sum_{i \in S} x_{i} \geq v_{m}(S)$ redundant. Then, there exists $\mathcal{B}_{2} \in \mathcal{F}_{k}^{m}(N \backslash S)$ such that

$$
\sum_{i \in S} x_{i}=\sum_{i \in N} x_{i}-\sum_{i \in N \backslash S} x_{i}=v(N)-\sum_{R \in \mathcal{B}_{2}} \gamma_{R}^{\mathcal{B}_{2}} \sum_{i \in R} x_{i} \geq v(N)-\sum_{R \in \mathcal{B}_{2}} \gamma_{R}^{\mathcal{B}_{2}} v_{D}(R) \geq v_{m}(S) \geq v(S) .
$$

Hence, it follows that $v(S) \leq v(N)-\min _{\mathcal{B} \in \mathcal{F}_{k}^{m}(N \backslash S)} \sum_{R \in \mathcal{B}} \gamma_{R}^{\mathcal{B}} v_{D}(R)$ and (7) follows.

To conclude, we show the "if" part. Assume that

$$
v(S) \leq \max \left\{\max _{\mathcal{B} \in \mathcal{F}_{k}^{m}(S)} \sum_{R \in \mathcal{B}} \gamma_{R}^{\mathcal{B}} v_{m}(R), v(N)-\min _{\mathcal{B} \in \mathcal{F}_{k}^{m}(N \backslash S)} \sum_{R \in \mathcal{B}} \gamma_{R}^{\mathcal{B}} v_{D}(R)\right\}
$$

for every $S \subseteq N$. We have to show that $\mathcal{C C}^{k}(v)=\mathcal{C}$ ore $(v)$. By Theorem 3.6, it suffices to prove that $\mathcal{C C}^{k}(v) \subseteq \mathcal{C}$ ore $(v)$. Let $x \in \mathcal{C C}^{k}(v)$, then,

$$
\sum_{i \in N} x_{i}=v(N)
$$

and $v_{m}(R) \leq \sum_{i \in R} x_{i} \leq v_{D}(R)$ for every $R \subseteq N$ with $|R| \leq k$. We have to show that $\sum_{i \in S} x_{i} \geq$ $v(S)$ for every $S \subseteq N$. 
First, let $S \subseteq N$ with $|S| \leq k$, then,

$$
\sum_{i \in S} x_{i} \geq v_{m}(S) \geq v(S)
$$

Second, let $S \subseteq N$ with $|S| \geq|N|-k$. Then, $|N \backslash S| \leq k$ and

$$
\sum_{i \in S} x_{i}=\sum_{i \in N} x_{i}-\sum_{i \in N \backslash S} x_{i}=v(N)-\sum_{i \in N \backslash S} x_{i} \geq v(N)-v_{D}(N \backslash S)=v(S)
$$

where the first inequality follows from the fact that $\sum_{i \in R} x_{i} \leq v_{D}(R)$ for every $R \subseteq N$ with $|R| \leq k$.

Third, let $S \subseteq N$ with $k<|S|<|N|-k$. We distinguish between two situations:

1. $\max _{\mathcal{B} \in \mathcal{F}_{k}^{m}(S)} \sum_{R \in \mathcal{B}} \gamma_{R}^{\mathcal{B}} v_{m}(R) \geq v(N)-\min _{\mathcal{B} \in \mathcal{F}_{k}^{m}(N \backslash S)} \sum_{R \in \mathcal{B}} \gamma_{R}^{\mathcal{B}} v_{D}(R)$.

By Condition (7), we have that $v(S) \leq \max _{\mathcal{B} \in \mathcal{F}_{k}^{m}(S)} \sum_{R \in \mathcal{B}} \gamma_{R}^{\mathcal{B}} v_{m}(R)$. Then, for $\overline{\mathcal{B}} \in \arg \max _{\mathcal{B} \in \mathcal{F}_{k}^{m}(S)} \sum_{R \in \mathcal{B}} \gamma_{R}^{\mathcal{B}} v_{m}(R)$, it follows that

$$
\sum_{i \in S} x_{i}=\sum_{R \in \overline{\mathcal{B}}} \gamma_{R}^{\overline{\mathcal{B}}} \sum_{i \in R} x_{i} \geq \sum_{R \in \overline{\mathcal{B}}} \gamma_{R}^{\overline{\mathcal{B}}} v_{m}(R)=\max _{\mathcal{B} \in \mathcal{F}_{k}^{m}(S)} \sum_{R \in \mathcal{B}} \gamma_{R}^{\mathcal{B}} v_{m}(R) \geq v(S),
$$

where the inequality follows from the fact that $\sum_{i \in R} x_{i} \geq v_{m}(R)$ for every $R \subseteq N$ with $|R| \leq k$.

2. $v(N)-\min _{\mathcal{B} \in \mathcal{F}_{k}^{m}(N \backslash S)} \sum_{R \in \mathcal{B}} \gamma_{R}^{\mathcal{B}} v_{D}(R)>\max _{\mathcal{B} \in \mathcal{F}_{k}^{m}(S)} \sum_{R \in \mathcal{B}} \gamma_{R}^{\mathcal{B}} v_{m}(R)$.

By Condition (7), we have that $v(S) \leq v(N)-\min _{\mathcal{B} \in \mathcal{F}_{k}^{m}(N \backslash S)} \sum_{R \in \mathcal{B}} \gamma_{R}^{\mathcal{B}} v_{D}(R)$. Then, for $\overline{\mathcal{B}} \in \arg \min _{\mathcal{B} \in \mathcal{F}_{k}^{m}(N \backslash S)} \sum_{R \in \mathcal{B}} \gamma_{R}^{\mathcal{B}} v_{D}(R)$, it follows that

$$
\begin{aligned}
\sum_{i \in S} x_{i}= & \sum_{i \in N} x_{i}-\sum_{i \in N \backslash S} x_{i}=v(N)-\sum_{R \in \overline{\mathcal{B}}} \gamma_{R}^{\overline{\mathcal{B}}} \sum_{i \in R} x_{i} \\
& \geq v(N)-\sum_{R \in \overline{\mathcal{B}}} \gamma_{R}^{\overline{\mathcal{B}}} v_{D}(R)=v(N)-\min _{\mathcal{B} \in \mathcal{F}_{k}^{m}(N \backslash S)} \sum_{R \in \mathcal{B}} \gamma_{R}^{\mathcal{B}} v_{D}(R) \geq v(S),
\end{aligned}
$$

where the inequality follows from the fact that $\sum_{i \in R} x_{i} \leq v_{D}(R)$ for every $R \subseteq N$ with $|R| \leq k$.

Note that, for the case $k=1$, we have that the characterization in Theorem 4.12 boils down to Theorem 2.2. 
Example 4.13. Consider the 6-player game $v \in G^{N}$ given by

$$
v(S)= \begin{cases}0 & \text { if }|S|=1, \\ 2 & \text { if }|S|=2, \\ 2 & \text { if }|S|=3, \\ 5 & \text { if }|S|=4 \\ 15 & \text { if }|S|=5, \\ 20 & \text { if }|S|=6 .\end{cases}
$$

It turns out that this game is 2-compromise stable, but not 1-compromise stable, that is, Core $(v) \neq$ $\mathcal{C C}^{1}(v)$ while $\mathcal{C}$ ore $(v)=\mathcal{C C}^{2}(v)=\mathcal{C}_{\text {ore }}{ }^{2}\left(v_{m}\right) \cap \mathcal{A C}$ ore $^{2}\left(v_{D}\right)$. In Table 2, we provide the values of $v$, $v_{D}, v_{m}$, together with the values of $\max _{\mathcal{B} \in \mathcal{F}_{2}^{m}(S)} \sum_{R \in \mathcal{B}} \gamma_{R}^{\mathcal{B}} v_{m}(R)$ and $v(N)-\min _{\mathcal{B} \in \mathcal{F}_{2}^{m}(N \backslash S)} \sum_{R \in \mathcal{B}} \gamma_{R}^{\mathcal{B}} v_{D}(R)$ for every $S \subseteq N$. It follows, by Theorem 4.12, that $\operatorname{Core}(v)=\mathcal{C C}^{2}(v)$. However, by Lemma 4.11 a) and b), we have that $\operatorname{Core}^{2}\left(v_{m}\right) \neq \operatorname{Core}(v)$ and $\mathcal{A C o r e}{ }^{2}\left(v_{D}\right) \neq \operatorname{Core}(v)$.

\begin{tabular}{|c|c|c|c|c|c|c|}
\hline$|S|$ & 1 & 2 & 3 & 4 & 5 & 6 \\
\hline$v(S)$ & 0 & 2 & 2 & 5 & 15 & 20 \\
\hline$v_{D}(S)$ & 5 & 15 & 18 & 18 & 20 & 20 \\
\hline$v_{m}(S)$ & 0 & 2 & 2 & 10 & 15 & 20 \\
\hline $\max _{\mathcal{B} \in \mathcal{F}_{2}^{m}(S)} \sum_{R \in \mathcal{B}} \gamma_{R}^{\mathcal{B}} v_{m}(R)$ & 0 & 2 & 3 & 4 & 5 & 8 \\
\hline$v(N)-\min _{\mathcal{B} \in \mathcal{F}_{2}^{m}(N \backslash S) \sum_{R \in \mathcal{B}} \gamma_{R}^{\mathcal{B}} v_{D}(R)}$ & -5 & 0 & 5 & 10 & 15 & 20 \\
\hline
\end{tabular}

Table 2: Game, utopia game, and minimal rights game in Example 4.13.

\section{Assignment games and 2-compromise stability}

This section shows that assignment games as introduced in Shapley (1967) are 2-compromise stable. In an assignment situation there is a two sided market with finite and disjoint set of buyers, $M$, and of sellers, $M^{\prime}$. We denote $m=|M|, m^{\prime}=\left|M^{\prime}\right|$, and $|N|=m+m^{\prime}$. The worth obtained when one buyer $i \in M$ and one seller $j \in M^{\prime}$ decide to cooperate is $a_{i j} \geq 0$. These values can be represented in an $m \times m^{\prime}$ matrix $A$. Following the notation in Núñez and Rafels (2002), a matching between coalitions $S \subseteq M$ and $T \subseteq M^{\prime}$ is a subset $\mu$ of $S \times T$ such that each player belongs to at most one pair in $\mu$. Given two coalitions $S \subseteq M$ and $T \subseteq M^{\prime}$, we denote the set of matchings between $S$ and $T$ by $\mathcal{M}(S, T)$; then, the maximum value that $S \cup T$ can obtain from cooperation is $\max _{\mu \in \mathcal{M}(S, T)} \sum_{(i, j) \in \mu} a_{i j}$.

Given an assignment situation $\left(\left(M, M^{\prime}\right), A\right)$, the associated assignment game $\left(M \cup M^{\prime}, v\right)$ is defined by

$$
v(S \cup T)=\max _{\mu \in \mathcal{M}(S, T)} \sum_{(i, j) \in \mu} a_{i j}
$$

Given an optimal matching $\mu \in \mathcal{M}\left(M, M^{\prime}\right)$ for $M$ and $M^{\prime}$, Shapley and Shubik (1972) show 
that the nonempty core of the assignment game $v$ is given by

$$
\operatorname{Core}(v)=\left\{\begin{array}{l|l}
(x, y) \in \mathbb{R}^{M \times M^{\prime}} & \begin{array}{l}
x_{i} \geq 0 \text { for all } i \in M, y_{j} \geq 0 \text { for all } j \in M^{\prime}, \\
x_{i}+y_{j}=a_{i j} \text { if }(i, j) \in \mu, \\
x_{i}+y_{j} \geq a_{i j} \text { if }(i, j) \notin \mu, \\
x_{i}=0 \text { if } i \text { is not assigned by } \mu, y_{j}=0 \text { if } j \text { is not assigned by } \mu
\end{array}
\end{array}\right\}
$$

Theorem 5.1. Assignment games are 2-compromise stable.

Proof. Let $\left(\left(M, M^{\prime}\right), A\right)$ be an assignment situation and let $\left(M \cup M^{\prime}, v\right)$ be the associated assignment game. It suffices to show that $\mathcal{C}$ ore $(v)=\mathcal{C C}^{2}(v)$. By Theorem 3.6, we know that $\operatorname{Core}(v) \subseteq \mathcal{C C}^{2}(v)$. Therefore, we only need to show that $\operatorname{Core}(v) \supseteq \mathcal{C C}^{2}(v)$. Let $\mu \in \mathcal{M}\left(M, M^{\prime}\right)$ be an optimal matching for $M$ and $M^{\prime}$, let $(x, y) \in \mathcal{C C}^{2}(v)$, and let $i \in M$ and $j \in M^{\prime}$. Clearly, $v_{m}(\{i\}) \leq x_{i} \leq v_{D}(\{i\})$ and $v_{m}(\{j\}) \leq y_{j} \leq v_{D}(\{j\})$ for every $i \in M$ and $j \in M^{\prime}$. Note that $v_{m}(\{i\}) \geq v(\{i\})=0$, and $v_{m}(\{j\}) \geq v(\{j\})=0$, thus,

$$
x_{i} \geq 0 \text { for all } i \in M \text { and } y_{j} \geq 0 \text { for all } j \in M^{\prime} .
$$

First, let $(i, j) \in \mu$. Then,

$$
v_{D}(\{i, j\})=v\left(M \cup M^{\prime}\right)-v\left((M \backslash\{i\}) \cup\left(M^{\prime} \backslash\{j\}\right)\right)=a_{i j}=v(\{i, j\}) \leq v_{m}(\{i, j\}) \leq v_{D}(\{i, j\})
$$

where the second equality follows because $\mu$ is optimal for $M$ and $M^{\prime}$ and $(i, j) \in \mu$. Therefore, all inequalities are equalities. Since $(x, y) \in \mathcal{C C}^{2}(v)$, we have that $a_{i j}=v_{m}(\{i, j\}) \leq x_{i}+y_{j} \leq$ $v_{D}(\{i, j\})=a_{i j}$ and

$$
x_{i}+y_{j}=a_{i j}
$$

Second, let $(i, j) \notin \mu$. Then, $v_{m}(\{i, j\}) \geq v(\{i, j\})=a_{i j}$. Since $x_{i}+x_{j} \geq v_{m}(\{i, j\})$, we have that

$$
x_{i}+y_{j} \geq a_{i j}
$$

Third, let $i \in M$ be not assigned by $\mu$. Then,

$$
v_{D}(\{i\})=v\left(M \cup M^{\prime}\right)-v\left((M \backslash\{i\}) \cup M^{\prime}\right)=0=v(\{i\}) \leq v_{m}(\{i\}) \leq v_{D}(\{i\})
$$

where the second equality follows because $\mu$ is optimal for $M$ and $M^{\prime}$ and $i$ is not assigned by $\mu$. Therefore, all inequalities are equalities. Since $(x, y) \in \mathcal{C C}^{2}(v)$, we have that $0=v_{m}(\{i\}) \leq$ $x_{i} \leq v_{D}(\{i\})=0$ and

$$
x_{i}=0
$$

Analogously, if $j \in M^{\prime}$ is not assigned by $\mu$, then, $v_{m}(\{j\})=0=v_{D}(\{j\})$ and

$$
y_{j}=0
$$


Therefore, $(x, y) \in \operatorname{Core}(v)$.

Remark 5.1. In fact, it can be seen that the core of an assignment game coincides with the 2core of the corresponding minimal rights game and with the 2-anti core of the utopia game. Therefore, the class of assignment games satisfies the conditions in Lemma $4.11 \mathrm{c}$ ).

The following example illustrates that assignment games can be 1-compromise stable.

Example 5.2. Consider $M=\{1,2,3\}$ and $M^{\prime}=\{4\}$ and the assignment matrix $A=(1,1,1)^{t}$. It is easy to check that $\operatorname{Core}(v)=\{(0,0,0,1)\}=\mathcal{C C}^{1}(v)$. This game is both 1-and 2-compromise stable.

Consider $M=\{1,2\}$ and $M^{\prime}=\{3,4\}$ and the assignment matrix

$$
A=\left(\begin{array}{ll}
5 & 3 \\
4 & 3
\end{array}\right)
$$

Then, $\operatorname{Core}(v)=\operatorname{con}\{(0,0,5,3),(1,0,4,3),(3,3,2,0),(4,3,2,1)\}=\mathcal{C C}^{2}(v)$ while $\operatorname{Core}(v) \neq \mathcal{C C}^{1}(v)$ since $(1,3,1,3) \in \mathcal{C C}^{1}(v) \backslash \mathcal{C}$ ore $(v)$. This game is 2-compromise stable, but not 1-compromise stable.

\section{Appendix}

In this Appendix, we provide a proof of Theorem 4.8. First, we recall the duality theorem.

Lemma A.1 (Duality theorem). Let $A \in \mathcal{M}_{m \times n}(\mathbb{R})$ be an $m \times n$ matrix, $b \in \mathbb{R}^{m}$, and $c \in \mathbb{R}^{n}$. If $\left\{x \in \mathbb{R}^{m}: x^{T} A \geq b^{T}\right\} \neq \varnothing$ and $\left\{y \in \mathbb{R}^{n}: A y=c, y \geq 0\right\} \neq \varnothing$, then,

$$
\min \left\{x^{T} c: x \in \mathbb{R}^{m}, x^{T} A \geq b^{T}\right\}=\max \left\{b^{T} y: y \in \mathbb{R}^{n}, A y=c, y \geq 0\right\}
$$

Proof of Theorem 4.8. Using the definition of the utopia game and the fact that every element of the $k$-core cover satisfies efficiency, we can reformulate the definition of the $k$-core cover as follows

$$
\mathcal{C C}^{k}(v)=\left\{\begin{aligned}
& \sum_{i \in R} x_{i} \geq v_{m}(R) \text { for all } R \subseteq N, 0<|R| \leq k \\
x \in \mathbb{R}^{N}: & \sum_{i \in R} x_{i} \geq v(R) \text { for all } R \subseteq N,|N|-k \leq|R|<|N| \\
& \sum_{i \in N} x_{i}=v(N)
\end{aligned}\right\} .
$$

Therefore, the $k$-core cover is the set of optimal solutions to the linear programming problem 
(P) given by

$$
\begin{aligned}
(P) \min & \sum_{i \in N} x_{i} \\
& \sum_{i \in R} x_{i} \geq v_{m}(R) \text { for every } R \subseteq N \text { with } 0<|R| \leq k, \\
& \sum_{i \in R} x_{i} \geq v(R) \text { for every } R \subseteq N \text { with }|N|-k \leq|R|<|N|, \\
& \sum_{i \in N} x_{i} \geq v(N)
\end{aligned}
$$

if the optimal value is $v(N)$. Note that the corresponding dual programming problem (D) is

$$
\begin{aligned}
& \text { (D) } \max \sum_{\substack{R \subseteq N: \\
0<|R| \leq k}} \delta_{R} v_{m}(R)+\sum_{\substack{R \subseteq N: \\
|N|-k \leq|R|<|N|}} \delta_{R} v(R)+\delta_{N} v(N)
\end{aligned}
$$

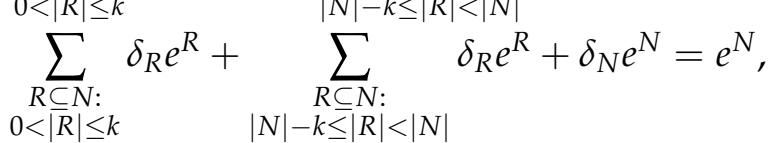

$$
\begin{aligned}
& \delta_{R} \geq 0 \text { for all } R \subseteq N \text { with } 0<|R| \leq k \text {, } \\
& \delta_{R} \geq 0 \text { for all } R \subseteq N \text { with }|N|-k \leq|R|<|N| \text {, } \\
& \delta_{N} \geq 0 \text {. }
\end{aligned}
$$

Obviously, the set of feasible solutions of $(\mathrm{P})$ is nonempty (we can take $x_{i}=a \in \mathbb{R}$ for every $i \in N$ with $a$ big enough so that $\sum_{i \in R} x_{i}=|R| a \geq v_{m}(R)$ for every $R \subseteq N$ with $0<|R| \leq k$, $\sum_{i \in R} x_{i}=|R| a \geq v(R)$ for every $R \subseteq N$ with $|N|-k \leq|R|<|N|$, and $\left.\sum_{i \in N} x_{i}=|N| a \geq v(N)\right)$. Moreover, the set of feasible solutions of (D) is nonempty (we can take $\delta_{N}=1$ and $\delta_{R}=0$ for every $R \subseteq N$ with either $0<|R| \leq k$, or $|N|-k \leq|R|<|N|)$. Besides, we have that $\mathcal{C C}^{k}(v) \neq \varnothing$ if, and only if, $v(N)$ is the optimal value of the linear programming problem $(\mathrm{P})$ which, by Lemma A.1, is true if, and only if, $v(N)$ is the optimal value of the dual programming problem (D). This is true if, and only if,

$$
v(N) \geq \sum_{\substack{R \in \mathcal{B}: \\ 0<|R| \leq k}} \delta_{R} v_{m}(R)+\sum_{\substack{R \in \mathcal{B}: \\|N|-k \leq|R|<|N|}} \delta_{R} v(R)+\delta_{N} v(N)
$$

for every $\mathcal{B} \in \mathcal{F}_{k,|N|-k}(N)$ and for every $\delta \in \Delta(\mathcal{B})$. Using the definition of utopia game, we can rewrite the equation above as

$$
v(N) \geq \sum_{\substack{R \in \mathcal{B}: \\ 0<|R| \leq k}} \delta_{R} v_{m}(R)-\sum_{\substack{R \in \mathcal{B} \\|N|-k \leq|R|<|N|}} \delta_{R} v_{D}(N \backslash R)+\sum_{\substack{R \in \mathcal{B} \\|N|-k \leq|R|<|N|}} \delta_{R} v(N)+\delta_{N} v(N)
$$

for every $\mathcal{B} \in \mathcal{F}_{k,|N|-k}(N)$ and for every $\delta \in \Delta(\mathcal{B})$.

To conclude the proof, we show that $v$ is $k$-compromise admissible if, and only if, it satisfies the inequalities for minimal balanced families on $N$ in $\mathcal{F}_{k,|N|-k}^{m}(N)$. Note that if a game is $k$ compromise admissible, then, it also satisfies the inequalities for minimal balanced families on 
$N$ in $\mathcal{F}_{k,|N|-k}^{m}(N)$. Therefore, we only have to show that if $v$ satisfies the inequalities for every minimal balanced family on $N$ in $\mathcal{F}_{k,|N|-k}^{m}(N)$, then, it is also $k$-compromise admissible. Assume that $v$ satisfies the inequalities for every minimal balanced family on $N$ in $\mathcal{F}_{k,|N|-k}^{m}(N)$, and let $\mathcal{B} \in \mathcal{F}_{k,|N|-k}(N) \backslash \mathcal{F}_{k,|N|-k}^{m}(N)$ and $\left\{\delta_{R}\right\}_{R \in \mathcal{B}} \in \Delta(\mathcal{B})$. Then, there exist $\mathcal{B}_{1}, \ldots, \mathcal{B}_{r} \in \mathcal{F}_{k}^{m}(N)$ and $t_{1}, \ldots, t_{r} \in(0,1)$ with $\sum_{l=1}^{r} t_{l}=1$ such that $\mathcal{B}=\cup_{l=1}^{r} \mathcal{B}_{l}$ and $\delta_{R}=\sum_{l \in\{1, \ldots, r\}: \mathcal{B}_{l} \ni R} t_{l} \gamma_{R}^{\mathcal{B}_{l}}$. If $N \notin \mathcal{B}$, then,

$$
\begin{aligned}
& \sum_{\substack{R \in \mathcal{B} \\
0<|R| \leq k}} \delta_{R} v_{m}(R)-\sum_{\substack{R \in \mathcal{B} \\
|N|-k \leq|R|<|N|}} \delta_{R} v_{D}(N \backslash R)+\sum_{\substack{R \in \mathcal{B} \\
|N|-k \leq|R|<|N|}} \delta_{R} v(N) \\
& =\sum_{\substack{R \in \mathcal{B}: \\
0<|R| \leq k}}\left(\sum_{\substack{l \in\{1, \ldots, r\}: \\
R \in \mathcal{B}_{l}}} t_{l} \gamma_{R}^{\mathcal{B}_{l}}\right) v_{m}(R) \\
& -\sum_{\substack{R \in \mathcal{B} \\
|N|-k \leq|R|<|N|}}\left(\sum_{\substack{l \in\{1, \ldots, r\}: \\
R \in \mathcal{B}_{l}}} t_{l} \gamma_{R}^{\mathcal{B}_{l}}\right) v_{D}(N \backslash R)+\sum_{\substack{R \in \mathcal{B} \\
|N|-k \leq|R|<|N|}}\left(\sum_{\substack{l \in\{1, \ldots, r\}: \\
R \in \mathcal{B}_{l}}} t_{l} \gamma_{R}^{\mathcal{B}_{l}}\right) v(N) \\
& =\sum_{l=1}^{r} t_{l}\left(\sum_{\substack{R \in \mathcal{B}_{l}: \\
0<|R| \leq k}} \gamma_{R}^{\mathcal{B}_{l}} v_{m}(R)-\sum_{\substack{R \in \mathcal{B}_{l}: \\
|N|-k \leq|R|<|N|}} \gamma_{R}^{\mathcal{B}_{l}} v_{D}(N \backslash R)+\sum_{\substack{R \in \mathcal{B}_{l}: \\
|N|-k \leq|R|<|N|}} \gamma_{R}^{\mathcal{B}_{l}} v(N)\right) \\
& \leq \sum_{l=1}^{r} t_{l}\left(\left(1-\sum_{\substack{R \in \mathcal{B}_{l}: \\
|N|-k \leq|R|<|N|}} \gamma_{R}^{\mathcal{B}_{l}}\right) v(N)+\sum_{\substack{R \in \mathcal{B}_{l}: \\
|N|-k \leq|R|<|N|}} \gamma_{R}^{\mathcal{B}_{l}} v(N)\right) \\
& =\sum_{l=1}^{r} t_{l} v(N)=v(N) \text {. }
\end{aligned}
$$

If $N \in \mathcal{B}$, let $\mathcal{B}_{r}=\{N\}$. Note that $\mathcal{B} \backslash\{N\} \in \mathcal{F}_{k,|N|-k}$ and $\left\{\delta_{R}^{\prime}\right\}_{R \in \mathcal{B} \backslash\{N\}} \in \Delta(\mathcal{B} \backslash\{N\})$ with $\delta_{R}^{\prime}=\frac{\delta_{R}}{1-\delta_{N}}$ for every $R \in \mathcal{B} \backslash\{N\}$. Then,

$$
\begin{aligned}
& \sum_{\substack{R \in \mathcal{B}: \\
0<|R| \leq k}} \delta_{R} v_{m}(R)-\sum_{\substack{R \in \mathcal{B} \\
|N|-k \leq|R|<|N|}} \delta_{R} v_{D}(N \backslash R)+\sum_{\substack{R \in \mathcal{B}: \\
|N|-k \leq|R|<|N|}} \delta_{R} v(N)+\delta_{N} v(N) \\
= & \left(1-\delta_{N}\right)\left(\sum_{\substack{R \in \mathcal{B} \backslash\{N\}: \\
0<|R| \leq k}} \delta_{R}^{\prime} v_{m}(R)-\sum_{\substack{R \in \mathcal{B} \backslash\{N\}: \\
|N|-k \leq|R|<|N|}} \delta_{R}^{\prime} v_{D}(N \backslash R)+\sum_{\substack{R \in \mathcal{B} \backslash\{N\}: \\
|N|-k \leq|R|<|N|}} \delta_{R}^{\prime} v(N)\right)+\delta_{N} v(N) \\
\leq & \left(1-\delta_{N}\right) v(N)+\delta_{N} v(N)=v(N)
\end{aligned}
$$

where the inequality follows from above. 


\section{References}

Aumann R., Maschler M. (1985) Game theoretic analysis of a bankruptcy problem from the Talmud. Journal of Economic Theory 36:195-213

Bondareva, O.N. (1963) Some applications of linear programming methods to the theory of cooperative games. Problemy Kibernitiki 10:119-139 (in Russian)

Gillies, D.B. (1953) Some theorems on $n$-person games. PhD thesis, Princeton University

Gillies, D.B. (1959) Solutions to general non-zero-sum games. Annals of Mathematical Studies $40,47-85$.

Núñez, M. and Rafels, C. (2002) The assignment game: the $\tau$-value. International Journal of Game Theory 31:411-422

O'Neill, M. (1982) A problem of rights arbitration from the Talmud. Mathematical Social Sciences 2, 345-371.

Potters, J., Poos, R., Muto, S., Tijs, S.H. (1989) Clan games. Games and Economic Behavior 1:275-293

Quant, M., Borm, P., Reijnierse, H., van Velzen, B. (2005) The core cover in relation to the nucleolus and the weber set. International Journal of Game Theory 33:491-503

Schmeidler, D. (1969) The nucleolus of a characteristic function game. SIAM Journal on applied mathematics 17: 1163-1170

Shapley, L.S. (1967) On balanced sets and cores. Naval Research Logistics Quarterly 14:453-460

Shapley, L.S. (1971) Cores of convex games. International Journal of Game Theory 1:11-26

Shapley, L.S. and Shubik, M. (1972) The assignment game I: the core. International Journal of Game Theory 1:111-130

Tijs, S. and Lipperts, F. (1982) The hypercube and the core cover of the $n$-person cooperative games. Cahiers du Centre d'Études de Recherche Opérationnelle 24:27-37

Weber, R.J. (1988) Probabilistic values for games. In: Roth, A. E. (Ed.), The Shapley value. Essays in honor of L. S. Shapley. Cambridge University Press, Cambridge, pp. 101-119. 